\title{
Late complications of robot-assisted radical cystectomy with totally intracorporeal urinary diversion
}

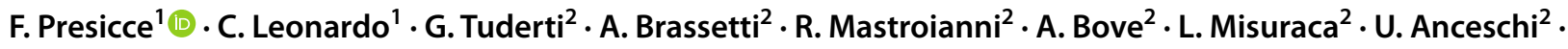 \\ M. Ferriero ${ }^{2} \cdot$ M. Gallucci ${ }^{2} \cdot G$. Simone ${ }^{2}$
}

Received: 27 May 2020 / Accepted: 20 July 2020 / Published online: 3 August 2020

(c) The Author(s) 2020

\begin{abstract}
Introduction and objectives To evaluate late complications in a large cohort of patients undergoing robot-assisted radical cystectomy (RARC) with totally intracorporeal urinary diversion (ICUD).

Materials and methods We prospectively enrolled patients who underwent RARC and ICUD between August 2012 and June 2019. We excluded patients with Ejection fraction $<36 \%$, retinal vasculopathy, ventriculoperitoneal shunts, and those treated without curative intent. All complications and their onset date have been recorded, defined, and graded according to Clavien classification adapted for radical cystectomy.

Results 210 patients were included, $76 \%$ of whom were men, with a mean age of 62 years. Urinary diversions used were Padua Ileal Bladder (PIB) in $80 \%$ of cases, and ileal conduit (IC) in $20 \%$ of patients (generally older and with more comorbidity). The mean follow-up was $30 \pm 22$ months. The stenosis rate of uretero-ileal anastomosis was $14 \%$, while a reduction in eGFR $(\geq 20 \%)$ was observed in about half of the cases. UTIs occurred in $37 \%$ of the patients, especially in the first 12 months. Only $2 \%$ of patients had bowel occlusion, whereas incisional hernia, lymphocele, and systemic events (metabolic acidosis and major cardiovascular events) occurred respectively in $20 \%, 10 \%$, and $1 \%$ of cases.

Conclusions Our study evaluates first late complications in a cohort of patients who underwent RARC with ICUD. These data are encouraging and in line with findings from a historical series of open radical cystectomy (ORC). This study is a further step in supporting RARC as a safe and effective surgical option for the treatment of muscle-invasive bladder cancer (MIBC) in tertiary referral centers.
\end{abstract}

Keywords Robotic cystectomy $\cdot$ Complications $\cdot$ Intracorporeal urinary diversion $\cdot$ Bladder cancer $\cdot$ Follow-up

\section{Introduction}

Open radical cystectomy (ORC) is still the standard treatment for MIBC and recurrent high-grade NMIBC [1]. However, over the last decade, robot-assisted radical cystectomy (RARC) has gradually gained popularity as a possible

Electronic supplementary material The online version of this article (https://doi.org/10.1007/s00345-020-03378-7) contains supplementary material, which is available to authorized users.

F. Presicce

fabriziopresicce@libero.it

1 Department of Urology, Sapienza - University of Rome, Via Alessandro Torlonia 12, Rome, Italy

2 Department of Urology, Regina Elena National Cancer Institute, Rome, Italy alternative therapeutic option [1]. In recent studies RARC has shown lower rate of intra- and peri-operative complications, with oncological results comparable to ORC, but with a longer operative time. In fact, recent evidence in the literature shows that RARC compared to ORC allows reduced blood losses and a shorter post-operative stay [2]. Furthermore, RARC has a lower rate of severe postoperative complications in the first ninety days of surgery [2]. Lastly, RARC would seem to provide oncological results comparable to ORC, at least in the short and medium-term [3-5].

However, although there is an increasing interest in RARC and several publications that have been published on this topic in recent years, it is not yet possible to draw definitive conclusions on the advantages and possible limitations of the robotic approach with respect to the open technique [1-5]. 
In fact, most of the data currently available derive from single-center series, with extracorporeal urinary derivation (ECUD) and with limited follow-up periods [1].

Furthermore, as also mentioned in the guidelines of the European Society of Urology (EAU), there are no available studies on late complications of RARC performed with totally intracorporeal urinary derivations (ICUD) [1].

Aim of our study was to evaluate, for the first time in the literature, late complications in a large cohort of patients undergoing RARC with totally intracorporeal urinary diversion (ICUD).

\section{Materials and methods}

\section{Design of the study}

We prospectively enrolled patients undergoing RARC and ICUD between August 2012 and June 2019.

All the data were inserted prospectively in a database approved by the institutional ethics committee and then retrospectively analyzed.

Inclusion criteria were muscle-invasive urothelial carcinoma of the bladder or recurrent high-grade nonmuscle-invasive bladder cancer refractory to intravesical immunotherapy.

Severe cardiovascular diseases with an ejection fraction $<36 \%$, retinal vasculopathy and the presence of a ventriculoperitoneal shunt were the only contraindications to robotic surgery.

Patients treated for non-curative purposes (cT4b, palliative or salvage cystectomy, recurrent disease after radiotherapy) were also excluded from the analysis.

Demographic characteristics of the patients at the baseline, clinical, perioperative, pathological, and functional data and the short and long-term complications were systematically collected.

\section{Surgical technique, perioperative assistance, and pathological assessment}

A rigorous multidisciplinary approach has been employed towards all patients with $\mathrm{BCa}$, which has led to a substantial increase in the use of neoadjuvant chemotherapy.

ERAS (Enhanced Recovery After Surgery) protocols have also been adopted since the end of 2012 [6].

RARC with totally intracorporeal urinary diversion was performed with a surgical technique replicating the principles of the open approach and was extensively described in previous works of our group [3].

\section{Follow-up schedule}

Follow-up was performed in accordance with the guidelines of the European Association of Urology (EAU) [1].

To exclude a recurrence of local and distant disease, urinary cytology and a chest/abdomen CT were performed every six months up to the third year and then annually thereafter.

During follow-up, a cystoscopy and/or a PET/CT scan may have been requested at the discretion of the physicians.

Periodically, an estimate of the glomerular filtration rate (eGFR) according to the CKD-EPI (Chronic Kidney Disease Epidemiology Collaboration) formula was finally recorded for each patient [7].

\section{Complications}

All complications and their onset date have been recorded, defined, and graded according to the Clavien classification based on 5 degrees and 11 domains adapted for radical cystectomy [8]. The Clavien system is, in fact, generally used to report complications in a defined interval, usually ninety days, for perioperative complications. Possible complications were collected at each follow-up visit and all patients enrolled in the study had completed monitoring for at least 90 days after surgery, as we considered all those that occurred after 90 days from surgery as late complications.

\section{Statistical analysis}

Medians with interquartile ranges and means with standard deviations were used to report continuous variables, frequencies, and proportions for categorical variables.

Kaplan-Meier method was used to plot survival curves; survival rates were computed at 1,2 and 5 years after surgery. The significance level was set to a $p$-value $<0.05$. The statistical analysis was performed using the statistical package for Social Science (SPSS v. 24.0; IBM, Somers, NY, USA).

\section{Results}

Overall, 210 consecutive patients were included. Most were male patients (76\%), with a mean age of $61 \pm 8.7$ years and a BMI of $25.6 \pm 3.7 \mathrm{~kg} / \mathrm{m}^{2}$. Neoadjuvant chemotherapy was performed in $26 \%$ of the cases and the mean number of lymph nodes (LN) removed for the procedure was $30 \pm 12$. The most commonly used urinary diversion was PIB ( $80 \%$ of the cases), while the remaining 
patients underwent IC (20\%). At baseline, $16 \%$ of patients had grade 3 or higher chronic kidney disease $(\mathrm{CKD} \geq 3)$, while pre-operative mean eGFR value in the enrolled population was $82 \mathrm{ml} / \mathrm{min}$ (Table 1).

Patients with IC compared to those who received a PIB were generally older ( $64.7 \pm 9.6$ vs $60.6 \pm 8.2$ years $)$, with greater comorbidities, with worse renal function (CKD $\geq 3$ in $24 \%$ vs $14 \%$ of cases) and a more advanced disease stage (disease stage $\geq \mathrm{pT} 3 \mathrm{a}$ in $36 \%$ vs $26 \%$ of cases) (Table 2 ).

Table 1 Characteristics of the study population at baseline*

\begin{tabular}{ll}
\hline Age (years) & $61 \pm 8.68$ \\
Gender (males/females) & $160(76 \%) / 50(24 \%)$ \\
BMI (kg/m $\left.{ }^{2}\right)$ & $25.6 \pm 3.7$ \\
Diabetes & $25 / 210(12 \%)$ \\
Hypertension & $77 / 210(37 \%)$ \\
Myocardial infarction & $24 / 210(11 \%)$ \\
Hydronephrosis & $33 / 210(18 \%)$ \\
Preoperative eGFR & $82 \pm 24$ \\
Preoperative creatinine (mg/dl) & $1.03 \pm 0.4$ \\
Preoperative CKD $\geq 3$ & $33 / 210(16 \%)$ \\
Derivation & \\
Ileal conduit & $43 / 210(20 \%)$ \\
Padua ileal bladder & $167 / 210(80 \%)$ \\
American Society Anaesthesiology (ASA) & \\
score & \\
1 & \\
2 & $27 / 210(12 \%)$ \\
3 & $147 / 210(68 \%)$ \\
4 & $36 / 210(20 \%)$ \\
Surgical indication & $0 / 210(0 \%)$ \\
BCG Failure** & \\
Primary MIBC & $120 / 210(57 \%)$ \\
Neoadjuvant chemotherapy & $90 / 210(43 \%)$ \\
Stage pTNM & $54 / 172 * * *(31 \%)$ \\
$\geq$ T3a & \\
pN+ & $59 / 210(21 \%)$ \\
Lymph nodes removed & $52 / 210(25 \%)$ \\
Positive surgical margins & $30 \pm 12$ \\
Operative time (minutes) & $19 / 210(9 \%)$ \\
Preoperative haemoglobin (gr/dl) & $315 \pm 71.2$ \\
Hospital stay (days) & $11.6 \pm 1.8$ \\
\hline
\end{tabular}

$M I B C$ muscle-invasive bladder cancer, $N M I B C$ not MIBC

*Data are reported as mean value \pm SD and $n(\%)$ respectively for continuous and categorical variables

**BCG failure category includes patients with recurrent high-grade NMIBC and patients with MIBC progression during follow-up

***Number of patients with cT2-T4a, cNOM0 bladder cancer potentially eligible for neoadjuvant chemotherapy
Mean follow-up was $30 \pm 22$ months, with about half of patients reaching at least 2 years of follow-up (Supplementary Fig. S1).

At 5 years, recurrence-free survival (RFS), cancerspecific survival (CSS) and overall survival (OS) were $57 \pm 5 \%, 59 \pm 5 \%$ and $57 \pm 5$, respectively \% (Supplementary Figs. S2-S4).

\section{Complications}

\section{Safety of the upper urinary tract}

During follow-up approximately $14 \%$ of patients experienced a stenosis of uretero-ileal anastomosis, without statistically significant differences between the two diversions (12\% vs $15 \%$ for IC and PIB, respectively). Despite the low rate of stenosis, a significant decline in eGFR was observed, in particular for patients who underwent IC.

Likewise, an increase in the rate of patients with a $C K D \geq 3$ was observed $(C K D \geq 3$ in $44 \%$ and $58 \%$ of patients underwent PIB and IC, respectively; Table 3).

\section{Stones and infective complications}

None of the patients with IC developed urinary stones compared to $8 \%$ of PIB group.

The distribution of the events was quite homogeneous over the years (Tables 3, Supplementary Table S4).

Symptomatic urinary tract infection has been recorded in approximately $37 \%$ of cases during follow-up, without statistically significant differences between the two diversions.

The vast majority of the events were recorded in the first year of follow-up (Table 3, Supplementary Table S4).

\section{Incisional hernia and gastrointestinal complications}

Bowel occlusion was a rare event (2\%), occurring exclusively during the first year of follow-up (Tables 3, Supplementary Table S4).

In about $20 \%$ of cases, an incisional hernia was observed, without statistically significant differences between the two diversions (Tables 3, Supplementary Table S4).

\section{Systemic events}

Metabolic acidosis and acute cardiovascular events were particularly rare (about 1\%) and observed exclusively during the first year of follow-up (Tables 3, Supplementary Table S4).

In almost $10 \%$ of patients a lymphocele has been reported, occurring mainly during the first 12 months after surgery (Tables 3, Supplementary Table SS4). 
Table 2 Characteristics of the study population at baseline according to the type of urinary derivation performed*

\begin{tabular}{llll}
\hline & IC & PIB & $p$ \\
\hline Numbers & 43 & 167 & \\
Age (years) & $64.7 \pm 9.6$ & $60.6 \pm 8.2$ & 0.002 \\
Gender (males/females) & $72 \% / 28 \%$ & $75 \% / 25 \%$ & 0.700 \\
BMI (kg/m ${ }^{2}$ ) & $26 \pm 3$ & $26 \pm 4$ & 0.465 \\
Diabetes & $7 / 43(16 \%)$ & $18 / 167(11 \%)$ & 0.001 \\
Hypertension & $17 / 43(40 \%)$ & $60 / 167(36 \%)$ & 0.730 \\
Myocardial infarction & $7 / 43(16 \%)$ & $17 / 167(10 \%)$ & 0.001 \\
Hydronephrosis & $8 / 43(18 \%)$ & $25 / 167(15 \%)$ & 0.001 \\
American Society.Anaesthesiology (ASA) score & & & \\
1 & $5 / 43(12 \%)$ & $22 / 167(13 \%)$ & 0.456 \\
2 & $29 / 43(68 \%)$ & $118 / 167(71 \%)$ & \\
3 & $9 / 43(20 \%)$ & $27 / 167(16 \%)$ & \\
4 & $0 / 43(0 \%)$ & $0 / 167(0 \%)$ & 0.001 \\
Neoadjuvant chemotherapy & $14 / 43(34 \%)$ & $40 / 167(24 \%)$ & \\
Stage pTNM & & & 0.001 \\
$\geq$ T3a & $16 / 43(37 \%)$ & $43 / 167(26 \%)$ & 0.001 \\
pN+ & $22 / 43(50 \%)$ & $30 / 167(18 \%)$ & 0.137 \\
Operative time (minutes) & $299 \pm 70$ & $320 \pm 71$ & 0.191 \\
Preoperative haemoglobin (gr/dl) & $14.5 \pm 2.3$ & $13.9 \pm 1.9$ & 0.576 \\
24 h post-operative haemoglobin (gr/dl) & $11.1 \pm 3.5$ & $10.5 \pm 2.5$ & 0.234 \\
Time to bowel (days) & $4.4 \pm 1.9$ & $4.7 \pm 2.1$ & 0.0013 \\
Hospital stay (days) & $10.6 \pm 3$ & $13.1 \pm 12$ & \\
Positive surgical margins & $9 / 43(21 \%)$ & $82.9 \pm 24$ & \\
Preoperative eGFR & $79.9 \pm 23$ & \\
Preoperative CKD $\geq 3$ & $10 / 43(24 \%)$ & $23 / 167(14 \%)$ & \\
\hline
\end{tabular}

$M I B C$ muscle invasive bladder cancer, $N M I B C$ not MIBC

*Data are reported as mean value $\pm \mathrm{SD}$ and $n(\%)$ respectively for continuous and categorical variables
Table 3 List of complications in the study population and relative incidences

\begin{tabular}{lllll}
\hline Complications & Total (210 pts) & IC (43 pts) & PIB (167 pts) & $p$ \\
\hline Lymphocele & $19(9 \%)$ & $7(16 \%)$ & $12(7 \%)$ & 0.113 \\
Incisional hernia & $42(20 \%)$ & $7(16 \%)$ & $35(21 \%)$ & 0.640 \\
Stones & $17(8 \%)$ & $0(0 \%)$ & $17(10 \%)$ & 0.047 \\
$\begin{array}{l}\text { Ureteroileal } \\
\text { stricture }\end{array}$ & $30(14 \%)$ & $5(12 \%)$ & $25(15 \%)$ & 0.658 \\
$\begin{array}{l}\text { UTI } \\
\text { Cardiovascular }\end{array}$ & $2(1 \%)$ & $0(0 \%)$ & $2(1 \%)$ & 0.956 \\
$\quad$ events & & $15(34 \%)$ & $63(38 \%)$ & 0.312 \\
$\begin{array}{l}\text { Metabolic acido- } \\
\quad \text { sis }\end{array}$ & $3(1 \%)$ & $1(2 \%)$ & $2(1 \%)$ & 0.435 \\
$\begin{array}{l}\text { Bowel occlusion } \\
\begin{array}{l}10 \% \text { eGFR } \\
\text { reduction }\end{array}\end{array}$ & $149(71 \%)$ & $37(87 \%)$ & $112(67 \%)$ & 0.001 \\
$\begin{array}{l}20 \% \text { eGFR } \\
\text { reduction }\end{array}$ & $109(52 \%)$ & $34(79 \%)$ & $75(45 \%)$ & 0.001 \\
\begin{tabular}{l} 
CKD $\geq 3 \mathrm{a}$ \\
\hline
\end{tabular} & $98(47 \%)$ & $25(58 \%)$ & $73(44 \%)$ & 0.001 \\
\hline
\end{tabular}

\section{Discussion}

It has now been 16 years since Menon et al. have described RARC procedure for the first time but it is especially in the last decade that this approach has become more widespread throughout the world [9].

At the same time, publications on this topic have also increased. In particular, numerous evidences demonstrated equivalence between RARC and ORC in terms of oncological results not only in the short term but also in the mediumlong term (5 years of follow-up) [1-5, 10].

Moreover, RARC is a safe procedure, with a complication rate in the first 30 and 90 days equal to or less than ORC, regardless of whether to perform a totally intracorporeal or extra urinary diversion [11]. Conversely, data on late complications of RARC have been lacking in the literature until now $[1,12]$.

The reason is probably twofold: first of all, RARC has been running for a short time and with low volumes in many centers, therefore, a sample size with adequate follow-up has not yet been reached to try to answer this question; moreover, there is probably an intrinsic difficulty in systematically 
collecting late complications, considering that even for the ORC there are not many data on this topic in the literature.

In fact, Clavien system has proved to be a very useful and practical tool for classifying and grading perioperative complications in a short, well-defined time interval of 30 or 90 days after surgery $[8,13]$.

Unfortunately, this system is less suitable for reporting late complications. This is because some complications can develop, as demonstrated by our results (Supplementary Table S4), in a time-dependent manner.

Therefore, it is important to keep this concept in mind because otherwise early complications are over-represented while late ones could be underrepresented.

To the best of our knowledge, this is the first study to report late complications in the medium term (about 30 months of follow-up) in a consecutive series of patients who underwent RARC with ICUD.

The results appear to be largely in line with the previous literature from open series on this topic.

In the Hautmann report, at 30 months the stenosis rate of ureteroileal anastomosis in patients who underwent neobladder surgery ranged between 5 and $15 \%$, depending on the anastomotic technique adopted [14].

Similarly, in the Studer series including more than 400 patients who underwent ORC with IC, the stricture rate of ureteroileal anastomosis was around $14 \%$ with a 100 months follow-up [15].

In our series, the ureteroileal stricture rate, using the split nipple technique, was $15 \%$ for PIB and $12 \%$ for IC with a mean follow-up of 30 months. Longer follow-up is required to properly assess the risk of developing stricture over time.

Renal function evaluated as eGFR also tends to decrease significantly over time both in the group of patients subjected to PIB and in those with a IC.

In our experience, in fact, a $10 \%$ decline in eGFR was observed in about $70 \%$ of patients and a $20 \%$ eGFR reduction in almost $50 \%$ of the cohort.

Similarly, Gershman et al., in a series of about 1300 patients who underwent ORC, observed an eGFR decline of $10 \%$ in about $73 \%$ of cases and $20 \%$ eGFR decline in $61 \%$ of patients at 5 years [16].

The rate of urinary tract infections (UTIs) in patients undergoing cystectomy is very variable depending on the parameter used to define them.

In the Studer series, only including UTIs requiring hospitalization, the percentage was $23 \%$ [15]. In the Hautmann cohort, recording UTIs associated with fever, the incidence was around 5\% [14]. In Wood series, including about 70 ORC and neo-bladder patients, $78 \%$ of asymptomatic bacteriuria and $8 \%$ of urosepsis were found, respectively [17]. In our cohort, $37 \%$ of symptomatic UTIs were recorded and, as in the open series, many events occurred early in the course of follow-up.
No conduit stones were recorded in patients who underwent IC in our study.

Similarly, Studer series had not reported any events in the first two years of follow-up, but the percentage rose to $20 \%$ after 5 years from the surgical procedure [15].

According to this time trend, $10 \%$ of our patients who underwent PIB experienced stones of the reservoir with a more marked increase in cases starting from the third year of follow-up.

The incidence of bowel occlusion after ORC was in the previous literature between 0.8 and $11 \%[14,18,19]$. In our series, we recorded a very low rate of bowel obstruction (around 2\%). These findings could be probably explained by the fact that a totally intracorporeal reconstruction, by limitedly mobilizing intestinal loops, can reduce the occlusive risk to a minimum.

Finally, in line with the literature from open series, the cases of severe metabolic acidosis were around $1 \%$ and above all in the first year of follow-up [14, 15, 18, 19].

In light of these results and from comparisons with the open series, our study first demonstrates that RARC with ICUD stands the test of time, providing sufficient quality standards in terms of late complications.

However, some limitations of our study should be acknowledged. First, a follow-up time of about 30 months, although of a certain temporal extension, may not be sufficient to intercept all late complications. In fact, as described in the literature, some complications are time-dependent and tend to occur even after a long time from the surgical procedure $[14,15]$. Therefore, a prospective systematic collection of complications is mandatory to assess any significant deviations from these preliminary findings in a longer follow-up interval. Furthermore, these data were collected in a tertiary center, with a high surgical volume, with a great surgical experience both with open and robotic procedures, proposing orthotopic neobladder in all cases in which there was no absolute contraindication. Consequently, these results could differ and not be applicable in centers with low surgical volume, with less robotic experience and with other selection criteria for RARC with orthotopic ICUD [20]. This bias is consistent with the wide use of ECUD in most actual RARC series. As a consequence, the two randomized controlled trials comparing open vs RARC only included ECUD [21, 22]. We would aim to provide more definitive outcomes of open RC vs RARC-ICUD with the ongoing randomized clinical trial (NCT03434132) at our Institution.

\section{Conclusions}

We first reported the incidence of late complications following RARC with ICUD. This procedure demonstrated to stand the test of time, being the risk of developing late 
complications in line with the historical series of ORC. Recent literature has already demonstrated the non-inferiority of RARC in terms of oncological and perioperative results. Therefore, our study is a further step in supporting RARC as safe and effective surgical option for the treatment of MIBC in tertiary referral centers.

Acknowledgements Open access funding provided by Università degli Studi di Roma La Sapienza within the CRUI-CARE Agreement. Declared none.

Author contributions Presicce F: protocol/project development, data analysis, manuscript writing. Leonardo C: protocol/project development, data analysis, manuscript editing. Tuderti G: data collection, manuscript editing. Brassetti A: data collection, manuscript editing. Mastroianni R: data collection, manuscript editing. Bove A: data collection, manuscript editing. Misuraca L: data management, data analysis, manuscript editing. Anceschi U: data analysis, manuscript editing. Ferriero M: data management, manuscript editing. Proietti F: data analysis, manuscript editing. Gallucci M: protocol/project development, manuscript editing. Simone G: protocol/project development, data management, data analysis, manuscript editing.

\section{Compliance with ethical standards}

\section{Conflict of interest Declared none.}

Ethical approval All procedures performed in the study involving human participants were in accordance with the ethical standards of the institutional research committee and with the 1964 Helsinki Declaration and its later amendments or comparable ethical standards.

Informed consent Informed consent was obtained from all individual participants/parents included in the study.

Open Access This article is licensed under a Creative Commons Attribution 4.0 International License, which permits use, sharing, adaptation, distribution and reproduction in any medium or format, as long as you give appropriate credit to the original author(s) and the source, provide a link to the Creative Commons licence, and indicate if changes were made. The images or other third party material in this article are included in the article's Creative Commons licence, unless indicated otherwise in a credit line to the material. If material is not included in the article's Creative Commons licence and your intended use is not permitted by statutory regulation or exceeds the permitted use, you will need to obtain permission directly from the copyright holder. To view a copy of this licence, visit http://creativecommons.org/licenses/by/4.0/.

\section{References}

1. Alfred Witjes J, Lebret T, Compérat EM et al (2017) Updated 2016 EAU guidelines on muscle-invasive and metastatic bladder cancer. Eur Urol 71(3):462-475

2. Novara G, Catto JW, Wilson T et al (2015) Systematic review and cumulative analysis of perioperative outcomes and complications after robot-assisted radical cystectomy. Eur Urol 67(3):376-401

3. Simone G, Papalia R, Misuraca L et al (2018) Robotic intracorporeal padua ileal bladder: surgical technique, perioperative, oncologic and functional outcomes. Eur Urol 73(6):934-940
4. Brassetti A, Cacciamani G, Anceschi U et al (2020) Longterm oncologic outcomes of robot- assisted radicalcystectomy (RARC) with totally intracorporeal urinary diversion(ICUD): a multicenter study. World J Urol 38(4):837-843

5. Simone G, Tuderti G, Misuraca L et al (2018) Perioperative and mid-term oncologic outcomes of robotic assisted radical cystectomy with totally intracorporeal neobladder: results of a propensity score matched comparison with open cohort from a singlecentre series. Eur J Surg Oncol 44:1432

6. Karl A, Buchner A, Becker A et al (2014) A new concept for early recovery after surgery for patients undergoing radical cystectomy for bladder cancer: results of a prospective randomized study. $\mathbf{J}$ Urol 191(2):335-340

7. Levey AS, Stevens LA, Schmid CH et al (2009) A new equation to estimate glomerular filtration rate. Ann Intern Med 150(9):604-612

8. Shabsigh A, Korets R, Vora KC et al (2009) Defining early morbidity of radical cystectomy for patients with bladder cancer using a standardized reporting methodology. Eur Urol 55(1):164-174

9. Menon M, Hemal AK, Tewari A et al (2003) Nerve-sparing robotassisted radical cystoprostatectomy and urinary diversion. BJU Int 92(3):232-236

10. Yuh B, Wilson T, Bochner B et al (2015) Systematic review and cumulative analysis of oncologic and functional outcomes after robot-assisted radical cystectomy. Eur Urol 67(3):402-422

11. Rai BP, Bondad J, Vasdev N et al (2019) Robotic versus open radical cystectomy for bladder cancer in adults. Cochrane Database Syst Rev 4(4):CD011903

12. Wilson TG, Guru K, Rosen RC et al (2015) Best practices in robot-assisted radical cystectomy and urinary reconstruction: recommendations of the Pasadena Consensus Panel. Eur Urol 67(3):363-375

13. De Nunzio C, Cindolo L, Leonardo C et al (2013) Analysis of radical cystectomy and urinary diversion complications with the Clavien classification system in an Italian real life cohort. Eur J Surg Oncol 39(7):792-798

14. Hautmann RE, de Petriconi RC, Volkmer BG (2011) 25 years of experience with 1,000 neobladders: long-termcomplications. J Urol 185(6):2207-2212

15. Madersbacher S, Schmidt J, Eberle JM et al (2003) The journal of urology 2003; long-term outcome of ileal conduit diversion. J Urol 169(3):985-990

16. Gershman B, Eisenberg MS, Thompson RH et al (2015) Comparative impact of continent and incontinent urinary diversion on long-term renal function after radical cystectomy in patients with preoperative chronic kidney disease 2 and chronic kidney disease 3a. Int J Urol 22(7):651-656

17. Wood DP Jr, Bianco FJ Jr, Pontes JE, Heath MA, DaJusta D (2003) Incidence and significance of positive urine cultures in patients with an orthotopic neobladder. J Urol 169(6):2196-2199

18. Gburek BM, Lieber MM, Blute ML (1998) Comparison of studer ileal neobladder and ileal conduit urinary diversion with respect to perioperative outcome and late complications. J Urol 160(3 Pt 1):721-723

19. Abol-Enein H, Ghoneim MA (2001) Functional results of orthotopic ileal neobladder with serous-lined extramural ureteral reimplantation: experience with 450 patients. J Urol 165(5):1427-1432

20. Brassetti A, Tuderti G, Anceschi U et al (2019) Combined reporting of surgical quality, cancer control and functional outcomes of robot-assisted radical cystectomy with intracorporeal orthotopic neobladder into a novel trifecta. Minerva Urol Nefrol 71(6):590-596

21. Bochner BH, Dalbagni G, Marzouk KH et al (2018) Randomized trial comparing open radical cystectomy and robot-assisted laparoscopic radical cystectomy: oncologic outcomes. Eur Urol 74(4):465-471 
22. Parekh DJ, Reis IM, Castle EP et al (2018) Robot-assisted radical cystectomy versus open radical cystectomy in patients with bladder cancer (RAZOR): an open-label, randomised, phase 3, non-inferiority trial. Lancet 391(10139):2525-2536
Publisher's Note Springer Nature remains neutral with regard to jurisdictional claims in published maps and institutional affiliations. 